\title{
Speech Acts, Categoricity, and the Meanings of Logical Connectives
}

\author{
Ole Thomassen Hjortland
}

\begin{abstract}
In bilateral systems for classical logic, assertion and denial occur as primitive signs on formulas. Such systems lend themselves to an inferentialist story about how truth-conditional content of connectives can be determined by inference rules. In particular, for classical logic there is a bilateral proof system which has a property that Carnap in 1943 called categoricity. We show that categorical systems can be given for any finite many-valued logic using $n$-sided sequent calculus. These systems are understood as a further development of bilateralism—call it multilateralism. The overarching idea is that multilateral proof systems can incorporate the logic of a variety of denial speech acts. So against Frege we say that denial is not the negation of assertion and, with Mark Twain, that denial is more than a river in Egypt.
\end{abstract}

\section{Introduction}

Logical inferentialism is the idea that the meaning of a logical constant is fixed by the inferential rules which govern its use. This thought, despite its casual formulationor perhaps because of its casual formulation-has been widespread among philosophers of logic. Of course, those who subscribe to logical inferentialism have endorsed very different precisifications of the core thesis. One of these, that the meaning of logical connectives is determined by formal rules of proof systems (e.g., in natural deduction or sequent calculus) we will call proof-theoretic semantics (PTS).

Mostly, the PTS literature has been focused on two questions.

(A) What are the constraints a set of inference rules must obey in order to be meaning determining?

(B) Will these constraints, appropriately set, entail a revision of logic?

Received April 3, 2012; accepted January 3, 2013

2010 Mathematics Subject Classification: Primary 03B50, 03B20

Keywords: logical inferentialism, logical constants, categoricity, proof theory, sequent calculus

C 2014 by University of Notre Dame $\quad 10.1215 / 00294527-2798700$ 
Question A was prompted by the introduction of the rogue connective tonk in Prior [22]. ${ }^{1}$ Subsequently there have been numerous replies offering proof-theoretic constraints that rule out tonk but which standard (nonrogue) connectives are supposed to satisfy, for example, conservativeness, harmony, normalization, the subformula property, definitional reflection, and so on. ${ }^{2}$

Philosophers like Prawitz [20], Dummett [6], and Tennant [37], [38] argue that these proof-theoretic constraints suggest that the inference rules for classical negation are semantically defective. Thinking of the inference rules as an implicit definition, we can say that the negation rules semantically misfire - they fail to determine the meaning of the logical constant. Intutionistic negation, on the other hand, is said to comply with the proof-theoretic standard. ${ }^{3}$ In response, others have insisted that the proposed trouble with classical logic is merely an artefact of the formal presentations, in particular, the type of proof system they are embedded in or the choice of classical inference rules. The case for classical logic is made in different variations in Weir [43], Milne [18], [19], and Read [23].

There is, however, a third question about logical inferentialism which I think is at least equally important:

(C) What is the nature of semantic content in PTS, and how is the semantic content determined by inference rules?

The language used by logical inferentialists is rarely helpful on this matter. It is frequently claimed that inference rules are meaning conferring (e.g., [6, p. 289], and [18, p. 50]), meaning constitutive (e.g., [38, p. 234]), sense conferring ([38, p. 229]), that the meaning of the connectives can be read off of the rules (see [6, p. 205]), or even that the rules should be equated with the meaning. Presumably, these different glosses on what determining meaning comes to are not equivalent. For our purposes, however, we will stick with the idea that inference rules fix or determine the meaning, and leave other glosses behind.

Anyone subscribing to PTS ought not only have a story about how meaning is determined by inference rules but also what is being determined. We need to give content to notions such as "meaning conferring" or "meaning constitutive." In what follows, I will investigate one avenue for treating these issues, broadly speaking an account of the semantic role of proof conditions. Deviating from the tradition, I want to couple PTS with a reification or propositionalization of meaning in the form of truth conditions. The guiding idea is that inference rules carve out the semantic content of logical constants. Inferentially speaking, it is a matter of reading off truth conditions from an inferential practice. But this is not much better than a metaphor. To make the idea precise in terms of PTS, I will investigate the relationship between truth values and semantic valuations on one side and proof-theoretic inference rules on the other. It will become apparent that there is a sense in which the rules induce sets of valuations, which in turn specify truth conditions for logical constants.

The idea that a logical constant can semantically misfire not because the inference rules are proof-theoretically defective but because they fail to specify the truthconditional content of the constant, was suggested in some early responses to Prior's tonk. Both Stevenson [36] and Wagner [42] offer analyses of tonk in terms of truth-functional shortcomings. Wagner also suggests that what is required is a distinction between the sense of a logical constant on one hand and its reference on the other, a move that is developed in more detail in Hodes [16]. With such a Fregean 
bifurcation of the "meaning" of logical constants, it is open to the inferentialist to adopt a more reconciliatory position in which both proof conditions and truth conditions play a role. More precisely_-helping ourselves to a term coined by Hodes-one can think of the inference rules as being sense-constitutive. The sense of the logical constant in turn determines the reference by specifying a truth condition.

These early attempts have received little attention compared to the proof-theoretic tradition. There are at least two reasons for this. First, Stevenson and Wagner's discussions appear to be limited to classical semantics. Their suggestions are about Boolean functions, and there is no indication that they can be extended to more general applications. Of course, if one is in the market for a defense of classical logic, perhaps their approach has some merit, but that is not the aim of the present paper. Far from it, I think the success of PTS depends precisely on it having a wide range of applications, not unlike model-theoretic semantics (MTS). There are more things in heaven and earth than the Boolean universe.

Second, we are not provided with any precise formulation of how the sense of a logical constant systematically determines reference. In fact, it will turn out that this matter is nontrivial, even in classical propositional logic. So, without a better account of how truth-conditional content is carved out by the rules, we have failed to bridge the gap between the two features of a logical constant's meaning.

I will start out in Section 1 by giving a formal characterization of the how proof rules induce truth conditions - via a categoricity condition. I show, using an example first given by Carnap, how categoricity fails for standard axiomatizations of classical logic. Then, in Section 2, I introduce bilateral systems with primitive speech act signs for assertion and denial. Following Smiley [35] and Rumfitt [28] I show how such systems are categorical for classical logic. In Section 3 I give the analogous result for multiple conclusion systems, before I proceed in Section 4 to extend the technique to multilateral systems with speech acts beyond assertion and denial. In Section 5 I show that multilateral systems can be formally represented using $n$-sided sequent calculi and prove that for any finite many-valued logic there is a categorical multilateral system.

\section{Categoricity}

The problem of uniquely determining truth conditions from proof rules can be traced back to Carnap [5]. Carnap observes that standard axiomatizations of propositional classical logic fail to uniquely specify the class of admissible valuations. ${ }^{4}$ In other words, it includes nonstandard interpretations of the logical connectives. In particular, Carnap shows that as far as the proof system is concerned, there is nothing that rules out a valuation in which everything is true. Needless to say, this wrecks the intended truth-conditional reading for the negation: For any $A$, both $A$ and $\neg A$ can come out true. The result is that there is no precise sense in which the proof system determines the truth conditions of the logical connectives in question.

In order to see how Carnap's problem works, we need to be a bit more precise. Let WFF be the set of well-formed formulas in some propositional language $\mathscr{L}$, and let $\mathcal{V}$ be a set of truth values, with $\mathscr{D} \subseteq \mathcal{V}$ the set of designated values.

Definition 2.1 (Valuation space) A valuation $v$ is a function from each member of WFF to a value in $\mathcal{V}$. We call the set of all such valuations $U$ the universe of valuations. A subset $V$ of $U$ is a valuation space. 
As an example, let us look at the language of propositional classical logic and its Boolean values, $\{1,0\}$, where 1 is the only designated value. Accordingly, a valuation is any function $v:$ WFF $\rightarrow\{1,0\}$. It is critical to note that $U$ includes valuations that are not in the set of admissible valuations, $V_{\mathrm{CPL}}$, for classical logic. In other words, $U$ contains nonstandard valuations. For example, any valuation $v^{\prime}$ such that $v^{\prime}(A)=1=v^{\prime}(\neg A)$ is not in $V_{\mathrm{CPL}}$, but it is in $U$. It should be clear that similar differences occur for every connective in the language $\mathscr{L}$.

If the formal semantics for classical logic allowed the above valuation $v^{\prime}$, there would be a sense in which we have failed to give the appropriate meaning to the (Boolean) negation. Put differently, if the inference rules are to specify the truth conditions, they had better screen off ill-behaved valuations like $v^{\prime}$. And, in fact, sometimes the standard inference rules succeed in doing precisely this. Take the standard inference rules for $\wedge$ :

$$
\frac{A \quad B}{A \wedge B}(I \wedge) \quad \frac{A \wedge B}{A}\left(E \wedge_{1}\right) \quad \frac{A \wedge B}{B}\left(E \wedge_{2}\right) .
$$

We can think of these inference rules as ordered pairs of a set of formulas and a single formula, for example, $\langle\{A, B\}, A \wedge B\rangle$ for $I \wedge$. More generally we have the following.

Definition 2.2 (Sequents) Let a sequent be an ordered pair $\langle\Gamma, A\rangle$, where $\Gamma$ is a set of formulas and $A$ a formula (of some set of WFFs). A valuation $v$ confirms (or, is a model of) a sequent $\langle\Gamma, A\rangle$ just in case whenever $v(\Gamma)=1$, we also have $v(A)=1$. Otherwise, $v$ falsifies the sequent.

Sometimes we will write a sequent in the familiar form $\Gamma \vdash A$ for convenience.

Definition 2.3 ( $V$-validity) Let $V$ be a valuation space. A sequent $\langle\Gamma, A\rangle$ is $V$-valid if every valuation $v \in V$ confirms it.

With these definitions in the background, we can think of sequents as constraints on a valuation space $V$. We can "read off" the first conjunction inference rule $I \wedge$ that, for every valuation $v$ in some valuation space, whenever $v(A)=1$ and $v(B)=1$, then $v(A \wedge B)=1$. Similarly, the second rule says (contrapositively) that, for every valuation $v$, whenever $v(A)=0$, then $v(A \wedge B)=0$; this corresponds for the third rule. The result is the standard truth function associated with $\wedge$ :

$$
f^{\wedge}(x, y)= \begin{cases}1 & \text { if } x=1 \text { and } y=1 \\ 0 & \text { otherwise }\end{cases}
$$

Crucially, these constraints are available without us antecedently knowing the truth function associated with $\wedge$. Although we do need to know something about the semantic framework, that is, what the truth values are and which ones are designated.

So far so good. The problem starts when we realize that other connectives do not share this property with conjunction. In fact, as Carnap discovered, the standard inference rules for disjunction $(\vee)$, implication $(\rightarrow)$, and negation $(\neg)$ all fail to provide exhaustive information about their respective classical truth functions. Take for example the standard $\vee$-rules:

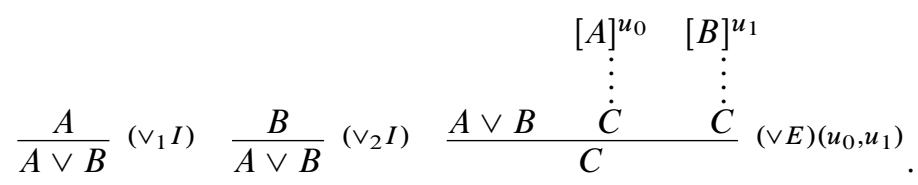


The leftmost rules $\vee I_{1}$ and $\vee I_{2}$ tell us that when $v(A)=1$, we have $v(A \vee B)=1$ and similarly for $v(B)=1$. Hence, we are only left with the task of determining the truth table line on which $v(A)=v(B)=0$. But the standard $\vee E$-rule underdetermines the matter: on the assumption that both disjuncts are false, we still cannot conclude that $A \vee B$ must be false as well. Why is this? The $\vee E$-rule does not schematize a sequent of the type $\langle\Gamma, A\rangle$ (i.e., from a set of formulas to a formula), rather, it only conditionally lets us infer $C$ from $A \vee B$, if there are already two independent subderivations of $C$ from $A$ and $C$ from $B .^{5}$

A natural conclusion to draw is that this limitation is caused by the fact that the only inferences we have considered are from a set of formulas to a formula, that is, sequents. Let us call this (proof-theoretic) framework \{FRML\}-FRML. As opposed to the case of conjunctions, both $\vee$ and $\rightarrow$ have rules that schematize inferences which are more complicated. In order to determine what their valuational contribution is, let us first formulate these rules in sequent-style natural deduction:

$$
\frac{\Gamma, A \vdash B}{\Gamma \vdash A \rightarrow B}(\rightarrow I) \quad \frac{\Gamma \vdash A \vee B \quad \Gamma, A \vdash C \quad \Gamma, B \vdash C}{\Gamma \vdash C}(\vee E) .
$$

Let us call this the framework of $\{$ SEQ $\}$-SEQ inferences, where $\{$ SEQ $\}$ is a set of sequents, and SEQ a sequent (whereas before the antecedent is a set of formulas, and the succedent a single formula). It is worth noticing that the \{FRML\}-FRML framework is simply a special case of the $\{$ SEQ $\}$-SEQ framework where the set of sequent premises is empty.

Since sequents are not true or false in a valuation, we need to give an alternative formulation of how such rules constrain the valuation space $V$.

Definition 2.4 A sequent rule $\{\mathrm{SEQ}\} \vdash \mathrm{SEQ}$ is $V$-valid just in case, for every valuation $v \in V$, whenever $v$ confirms every sequent in $\{\mathrm{SEQ}\}$ (the premise sequents), then $v$ confirms the conclusion sequent SEQ.

We can take $\rightarrow I$ as an example. Let $v(\Gamma)=1$, and let $v(A \rightarrow B)=0$. Then $v$ cannot confirm every premise sequent, so, since $v(\Gamma)=1, v(A)=1$ and $v(B)=0$. Thus we have that whenever $A \rightarrow B$ is false, $A$ is true and $B$ is false, from which it follows that if both $A$ and $B$ are false, then $A \rightarrow B$ is true, as is required for the target truth function.

What about $\vee E$ ? Again, the only problematic input in the truth function is when both $A$ and $B$ are false. First, take the instance where $C=A$. Again, assume that $v(\Gamma)=1$ and $v(A)=v(B)=0$. Then $\Gamma \vdash A$ is falsified, so $v$ cannot model all the premise sequents. Since $v(A)=v(B)=0$, both minor premise sequents are confirmed. Thus, $A \vee B$ must be false in the major premise, in order for $v$ to confirm the inference rule.

Negation also runs into problems in the $\{$ FRML $\}$-FRML framework. Consider, for instance, the following inference rules:

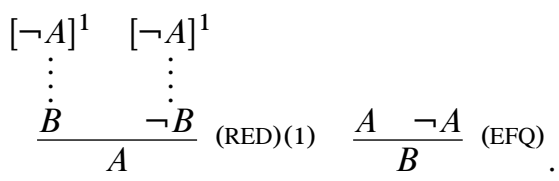

The EFQ-rule does not involve any subderivations, but it is not on its own adequate to determine the truth function for $\neg$. It excludes any valuation $v$ for which 
$v(A)=v(\neg A)=1$ and $v(B)=0$, but, importantly, it allows a valuation $v^{*}$ where $v^{*}(A)=1$, for every $A \in \mathrm{WFF}$. Needless to say, the valuation $v^{*}$ does not respect the truth function for $\neg$. It does, however, respect the truth functions for $\wedge, \vee$, and $\rightarrow$.

Can the $\{\mathrm{SEQ}\}-\mathrm{SEQ}$ framework improve the situation? None of the rules in the $\{$ SEQ $\}-S E Q$ framework we have looked at excludes the nonstandard valuation $v^{*}$. Actually, it is easy to see that no inference rule in the $\{$ SEQ\}-SEQ framework is falsified by the valuation $v^{*}$. It suffices to note that for each inference rule, the conclusion sequent will always be confirmed by $v^{*}$, and thus the valuation also confirms the inference rule.

We can now give a concrete example of noncategoricity. Let us take the following \{SEQ\}-SEQ system for classical logic. ${ }^{6}$

Definition 2.5 We have classical propositional logic, Ncp:

$$
\begin{aligned}
& A \vdash A \frac{\Gamma \vdash C}{\Gamma, A \vdash C}(K) \\
& \frac{\Gamma \vdash A \quad \Gamma \vdash B}{\Gamma \vdash A \wedge B}(\wedge I) \quad \frac{\Gamma \vdash A \wedge B}{\Gamma \vdash A}(\wedge E a) \quad \frac{\Gamma \vdash A \wedge B}{\Gamma \vdash B}(\wedge E b) \\
& \frac{\Gamma \vdash A \quad \Gamma \vdash B}{\Gamma \vdash A \wedge B}(\wedge I) \quad \frac{\Gamma \vdash A \wedge B}{\Gamma \vdash A}(\wedge E a) \quad \frac{\Gamma \vdash A \wedge B}{\Gamma \vdash B}(\wedge E b) \\
& \frac{\Gamma \vdash A \vee B \quad \Gamma, A \vdash C \quad \Gamma, B \vdash C}{\Gamma \vdash C}(\vee E) \frac{\Gamma \vdash A}{\Gamma \vdash A \vee B}(\vee I a) \frac{\Gamma \vdash B}{\Gamma \vdash A \vee B}(\vee I b) \\
& \frac{\Gamma, \neg A \vdash B \quad \Gamma, \neg A \vdash \neg B}{\Gamma \vdash A} \text { (RED) } \frac{\Gamma \vdash A \quad \Gamma \vdash \neg A}{\Gamma \vdash B} \text { (EFQ) }
\end{aligned}
$$

What we know from the above is that Nep, and in general any classical \{SEQ\}-SEQ system, is sound and complete with respect to at least two valuation spaces, that is, $V_{\mathrm{CPL}}$ and $V_{\mathrm{CPL}} \cup\left\{v^{*}\right\}$. This is the sense in which we have noncategoricity. There is no unique semantics for the connectives determined by the inference rules. On the latter valuation space, the nonstandard valuation violates the intended interpretation. ${ }^{7}$

Before we look at options for regaining categoricity, let us introduce some helpful definitions. ${ }^{8}$

Definition 2.6 Let a logic $L$ be a set of $\{$ SEQ $\}$-SEQ inferences (with respect to some language and set of WFFs). We say that an inference is $L$-valid if it is in $L$.

A proof system $S$ consisting of $\{$ SEQ $\}-S E Q$ inference rules and axioms (e.g., Ncp) will determine a corresponding set of inferences $L_{S}$, that is, the class of inferences provable in $S$. For instance, Ncp determines $L_{\mathrm{Ncp}}$, and for each inference $\{\mathrm{SEQ}\}-\mathrm{SEQ}$ provable in Ncp, we have $\{\mathrm{SEQ}\}-\mathrm{SEQ} \in L_{\mathrm{Ncp}}$.

We can then define the required interaction between $L$ and $V$.

Definition 2.7 For a valuation space $V \subseteq U$ and a logic $L$ :

$$
\mathbb{L}(V)={ }_{d f}\{\{\text { SEQ }\} \text {-SEQ } \in L:\{\text { SEQ }\} \text {-SEQ is } V \text {-valid }\} .
$$

Definition 2.8 ( $L$-consistency) Let $L$ be a logic, and let $v \in U$ be a valuation:

$v$ is $L$-consistent if and only if $v$ confirms every inference in $L$;

$\mathbb{V}(L)={ }_{d f}\{v \in U: v$ is $L$-consistent $\}$.

The pair $\langle\mathbb{L}, \mathbb{V}\rangle$ is an antitone Galois connection between the power set of $U$, ordered by inclusion, and the power set of $L$, ordered by inclusion, respectively. That is, for any $V \subseteq U$ and $L$ (for a given WFF), $L \subseteq \mathbb{L}(V)$ if and only if $V \subseteq \mathbb{V}(L)$.

We then have the following facts. 
Fact 1 For every valuation space $V$ and $\operatorname{logic} L$, respectively:

(i) $L \subseteq \mathbb{L}(\mathbb{V}(L))$,

(ii) $V \subseteq \mathbb{V}(\mathbb{L}(V))$.

Proof For (ii), assume for reductio that $V \nsubseteq \mathbb{V}(\mathbb{L}(V))$. Since $\langle\mathbb{L}, \mathbb{V}\rangle$ is an antitone Galois connection, it follows by contraposition that $\mathbb{L}(V) \nsubseteq \mathbb{L}(V)$.

But what about the converse? This is the question that will occupy us in what follows.

Definition 2.9 (Categoricity) Let $L$ be a logic, and let $V$ be a valuation space.

If $L=\mathbb{L}(\mathbb{V}(L))$, say that $L$ is categorical.

If $V=\mathbb{V}(\mathbb{L}(V))$, say that $V$ is categorical.

Our notion of categoricity is what Dunn and Hardegree calls absoluteness and what in general is called Galois completeness. ${ }^{9,10}$ Our focus is the categoricity of a valuation space $V$. If $V$ is the set of intended interpretations (standard valuations), then categoricity ensures that the inferences that are confirmed by every member of $V$ in turn have no $L$-consistent valuation $v$ that is nonstandard. It is thus straightforward to see that $V_{\mathrm{CPL}}$ is noncategorical, provided that the inferences induced by $\mathbb{L}$ are in the framework $\{$ FRML $\}$-FRML or $\{$ SEQ $\}-S E Q$. Simply observe that our nonstandard valuation $v^{*}$ is not in $V_{\mathrm{CPL}}$ but is in $\mathbb{V}\left(\mathbb{L}\left(V_{\mathrm{CPL}}\right)\right) .{ }^{11}$ The informal gloss on the noncategoricity result is that standard formalizations of classical logic fail to uniquely determine the semantic content of the logical constants. Nevertheless, there is no need to despair quite yet-the inferentialist has other resources.

\section{Unilateralism and Bilateralism}

One strategy for devising categorical systems is to introduce primitive speech act signs in the proof systems. This technique was first used to give a categorical system for classical logic in Smiley [35]. The idea was further elaborated by Rumfitt [28], with an emphasis on PTS. ${ }^{12}$ He proposes a system for classical logic using primitive signs in the formal language,,+- , interpreted as assertion and denial, respectively. ${ }^{13}$ Accordingly, Rumfitt's inference rules operate on signed formulas, $+A,-A$, read informally as " $A$ ? Yes" and " $A$ ? No," where $A$ is a formula in the language of classical logic. Rumfitt calls this type of system bilateral, as opposed to the standard unilateral natural deduction system where primitive denial is absent. In the background, of course, is the Frege-Geach debate about whether or not denial can be appropriately expressed as the assertion of a negated proposition. What Smiley and Rumfitt's work shows is that there is at least one advantage to bilateralism, namely, categoricity.

Using the signs + and - we can, for example, dualize the standard inference rules for disjunction, $I \vee$ and $E \vee$ (where $C$ can take either sign):

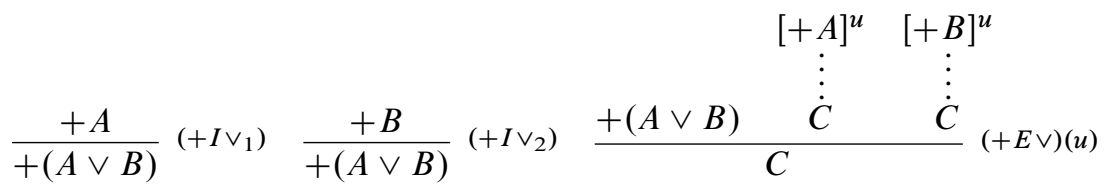

to acquire rules which are symmetric to the conjunction rules, $I \wedge$ and $E \wedge$ :

$$
\frac{-(A \vee B)}{-A}\left(-E \vee_{i}\right) \frac{-(A \vee B)}{-B}\left(-E \vee_{i i}\right) \frac{-A-B}{-(A \vee B)}(-I \vee)
$$


Correspondingly, the conjunction has disjunction-like rules for the denial sign in addition to the standard rules:

$$
\begin{aligned}
& \frac{+(A \wedge B)}{+A}\left(+E \wedge_{i}\right) \quad \frac{+(A \wedge B)}{+B}(+E \wedge i i) \frac{+A+B}{+(A \wedge B)}(+I \wedge) \\
& {[-A]^{u} \quad[-B]^{u}}
\end{aligned}
$$

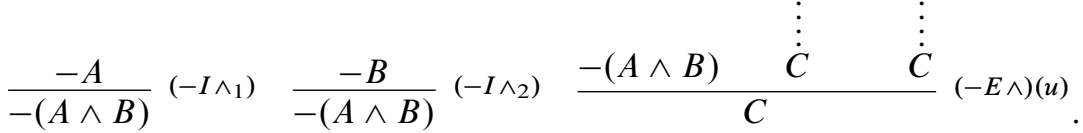

In fact, with the one proviso that the rules are embedded in a system containing two coordination principles for + and - , the result is a system where every inference rule is immediate, that is, involves no discharge of assumptions. As we should expect then, the coordination principles will compensate for this by themselves, reintroducing hypothetical reasoning:

$$
\begin{aligned}
& {[\alpha]^{u}} \\
& \vdots \\
& \frac{\dot{\perp}}{\alpha^{*}}\left(\mathrm{RED}^{*}\right)(u) \quad \frac{\alpha \quad \alpha^{*}}{\perp}\left(\mathrm{LNC}^{*}\right) .
\end{aligned}
$$

In $\mathrm{RED}^{*}$ and $\mathrm{LNC}^{*}$ we use $\alpha$ as a metavariable over signed formulas, with * indicating that the sign is reversed (i.e., + to - and - to + ). In other words, thinking of - as an unembeddable negation, RED* mimics both intuitionistic and classical reductio. The two rules are both valid in classical logic, but only the leftmost in intuitionistic logic:

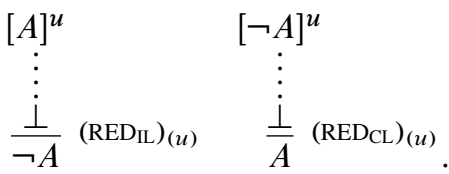

It is of special interest to the revisionary debate between classical and intuitionistic logic that Rumfitt's system allows us to simplify the classical negation rules:

$$
\frac{-A}{+(\neg A)}(+I \neg) \quad \frac{+(\neg A)}{-A}(+E \neg) \text {. }
$$

The two corresponding rules, $-I \neg$ and $-E \neg$, are derivable in the presence of the coordination rules. True, the new rules are only made available by shifting the burden of the problematic inferences - namely, classical reductio-over to the coordination principles. Yet, as Rumfitt insists, these inference rules are not governing the inferential practice of negation specifically, so the classicist and the intuitionist are endorsing the same basic rules of negation. Their disagreement only arises in the context of assertion and denial.

With the classical logic embedded in an enriched language with primitive assertion and denial, the system has properties corresponding to those of standard intuitionistic logic. Note for instance that there is a straightforward conversion transformation for Rumfitt's negation rules:

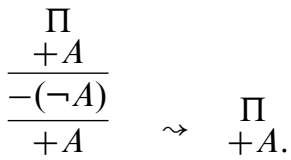


Here $\Pi$ is a subderivation whose end node is the premises for concluding $+A$. In fact, unlike standard axiomatizations of classical logic, the system is separable; that is, any proof $\Gamma \vdash A$ only requires inference rules for connectives occurring in a formula in $\Gamma$ or in $A$ (in addition to the coordination rules). ${ }^{14}$

In order to investigate a bilateral system with respect to categoricity, we will introduce a new proof-theoretic framework where inference rules are still in $\{$ SEQ $\}-S E Q$, but sequents are built up from signed formulas. Interestingly, the speech act signs make a crucial difference to how the inference rules can be read as constraints on a valuation space. I will use SEQ* to indicate a (single-conclusion) sequent of signed formulas and call the framework $\left\{\mathrm{SEQ}^{*}\right\}-\mathrm{SEQ}^{*}$, that is, inferences from a set of signed sequents to a single signed sequent.

Let us reformulate some of the bilateral inference rules in sequent-style natural deduction. Here is disjunction again (with $\Gamma^{*}$ a set of signed formulas):

$$
\frac{\Gamma^{*} \vdash-(A \vee B)}{\Gamma^{*} \vdash-A} \quad \frac{\Gamma^{*} \vdash-(A \vee B)}{\Gamma^{*} \vdash-B} \quad \frac{\Gamma^{*} \vdash-A \Gamma^{*} \vdash-B}{\Gamma^{*} \vdash-(A \vee B)} .
$$

Bilateral inference rules are not governing the sort of inferences that preserve truth (or designated-value-in-a-valuation). The reason is simple: signed formulas do not represent an expression of a proposition, they represent an expression of a judgment, that is, speech acts (e.g., + ) operating on a content (e.g., $A$ ). These speech acts are not the sort of things that are true or false, and thus accordingly, what is preserved from premises to conclusion in a bilateral inference is not truth but correctness. In short, $-A$ is correct just in case $A$ is false, and $+A$ is correct just in case $A$ is true. This might be a rather pedestrian formulation of the norms of assertion and denial, but we bracket such complications for now.

Definition 3.1 (Correctness) Let $\Gamma^{*}$ be a set of signed formulas, let $A^{*}$ be a signed formula, and let $v$ be a valuation. We say that $+A(-A)$ is correct on $v$ just in case $v(A)=1(v(A)=0)$. A bilateral sequent $\Gamma^{*} \vdash A^{*}$ is confirmed by a valuation $v$ just in case whenever $B^{*}$ is correct on $v$, for every $B^{*} \in \Gamma^{*}, A^{*}$ is correct on $v$.

Definition 3.2 ( $V$-validity) A $\left\{\mathrm{SEQ}^{*}\right\}-\mathrm{SEQ}^{*}$ sequent is $V$-valid just in case every $v \in V$ confirms it.

Definition 3.3 Let a (signed) $\operatorname{logic} L^{*}$ be a set of $\left\{\mathrm{SEQ}^{*}\right\}-\mathrm{SEQ}^{*}$ inferences (with respect to some language and set of WFFs). We say that an inference is $L^{*}$-valid if it is in $L^{*}$.

Definition 3.4 Let $V$ be a valuation space, and let $L^{*}$ be a logic:

$$
\mathbb{L}^{*}(V)={ }_{d f}\left\{\left\{\mathrm{SEQ}^{*}\right\}-\mathrm{SEQ}^{*} \in L^{*}:\left\{\mathrm{SEQ}^{*}\right\}-\mathrm{SEQ}^{*} \text { is } V \text {-valid }\right\} .
$$

The new pair $\left\langle\mathbb{L}^{*}, \mathbb{V}\right\rangle$ is also an antitone Galois connection between the power set of $U$, ordered by inclusion, and the power set of $L^{*}$, ordered by inclusion, respectively.

We can now prove the first theorem about valuation spaces $V$ (and $V_{\mathrm{CPL}}$ in particular) with respect to $\mathbb{L}^{*}$.

Theorem 3.5 (Smiley [35]) For any valuation space $V \subseteq U, V=\mathbb{V}\left(\mathbb{L}^{*}(V)\right)$.

Proof We only prove one direction; the other direction follows from the fact that the we are dealing with a Galois connection. The proof proceeds by contraposition. 
Let $v_{0} \notin V$. Define the sets $T, F$ as follows:

$$
\begin{aligned}
& T=\left\{A \in \mathrm{WFF}: v_{0}(A)=1\right\}, \\
& F=\left\{A \in \mathrm{WFF}: v_{0}(A)=0\right\} .
\end{aligned}
$$

Obviously, $T \cup F=W F F$. There are two cases: (i) $F=\emptyset$; and (ii) $F$ is nonempty. We show the first case and indicate the structure of the other three.

Case (i). Take the sequent $+(T / Q) \vdash-Q$, where $+(T / Q)$ is the set of + -signed formulas in $T$ except the formula $Q$. For each $v \neq v_{0}$, there will be some $B \in \mathrm{WFF}$ such that $v(B)=0$. Thus, either $v(Q)=0$ or, for some $B \in T / Q, v(B)=0$. That is, either some $+B \in+(T / Q)$ is incorrect or $-Q$ is correct. Either way, $v$ confirms the sequent $+(T / Q) \vdash-Q$. So, the argument is $V$-valid, and thus in $\mathbb{L}^{*}(V)$. Yet, as is evident, $v_{0}$ does not confirm $+(T / Q) \vdash-Q$, hence $v_{0}$ is not $\mathbb{L}^{*}(V)$-consistent. In other words, $v_{0} \notin \mathbb{V}\left(\mathbb{L}^{*}(V)\right)$.

Case (ii). Take the sequent $+T \cup\left\{-A_{0},-A_{1}, \ldots\right\} \vdash+Q$, where $F=\left\{Q, A_{0}\right.$, $\left.A_{1}, \ldots\right\}$. The technique is as before.

The proof of Theorem 3.5 actually only needs the degenerate case of $\left\{\mathrm{SEQ}^{*}\right\}-\mathrm{SEQ}^{*}$ inferences where $\left\{\mathrm{SEQ}^{*}\right\}=\emptyset$. In other words, we are only relying on a weaker framework of single signed sequents, that is, a set of signed formulas and a signed formula, $\left\{\mathrm{FRML}^{*}\right\}$-FRML ${ }^{*}$.

Let us look at an example. Recall the nonstandard valuation $v^{*}$ where $v^{*}(A)=1$, for every $A \in W F F$, which caused problems for Ncp. In a signed system (with the coordination rules) we can derive the sequent $+A \vdash-\neg A$. Thus, for every $v \in V$, if $+A$ is correct on $v$, then $-\neg A$ is also correct. That is to say, if $v(A)=1(0)$, then $v(\neg A)=0(1)$, which is what we require.

\section{Multiple Conclusion}

Smiley [35] shows that multiple conclusion sequents also yield categoricity for classical propositional logic.

Definition 4.1 (Multiple conclusion sequents) Let a multiple conclusion sequent be an ordered pair $\langle\Gamma, \Delta\rangle$ where both $\Gamma$ and $\Delta$ are (possibly empty) sets of formulas (of some set of $W F F \mathrm{~s}$ ). A valuation $v$ confirms a multiple conclusion sequent $\langle\Gamma, \Delta\rangle$ just in case whenever $v(\Gamma)=1$, we also have $v(B)=1$, for some $B \in \Delta$. Otherwise, $v$ falsifies the sequent.

As before, we will sometimes write the sequents in the familiar form $\Gamma \vdash \Delta$ for convenience. A multiple conclusion sequent inference will be an inference from a set of (multiple conclusion) sequents to a single sequent. We call this framework \{MSEQ\}-MSEQ.

Definition 4.2 ( $V$-validity) A multiple conclusion sequent $\langle\Gamma, \Delta\rangle$ is $V$-valid just in case it is confirmed by every $v \in V$.

Definition 4.3 Let a (multiple conclusion) logic $L^{M}$ be a set of \{MSEQ\}-MSEQ inferences (with respect to some language and set of $W F F \mathrm{~s}$ ). We say that an inference is $L^{M}$-valid if it is in $L^{M} \cdot{ }^{15}$

Definition 4.4 ( $L^{M}$-consistency) Let $L^{M}$ be a logic, and let $v \in U$ be a valuation:

$v$ is $L^{M}$-consistent if and only if $v$ confirms every inference in $L^{M}$. 
Definition 4.5 Let $V$ be a valuation space, and let $L^{M}$ be a logic:

$$
\begin{aligned}
& \mathbb{L}^{M}(V)={ }_{d f}\left\{\{\text { MSEQ }\} \text {-MSEQ } \in L^{M}:\{M S E Q\} \text {-MSEQ is } V \text {-valid }\right\}, \\
& \mathbb{V}\left(L^{M}\right)={ }_{d f}\left\{v \in U: v \text { is } L^{M} \text {-consistent }\right\} .
\end{aligned}
$$

Theorem 4.6 (\{MSEQ\}-MSEQ, Dunn and Hardegree [8]) For any valuation space $V \subseteq U, V=\mathbb{V}\left(\mathbb{L}^{M}(V)\right)$.

Proof Suppose that $v_{0} \notin V$. We show that $v_{0} \notin \mathbb{V}\left(\mathbb{L}^{M}(V)\right)$. Define two sets $T$, $F$ as follows:

$$
\begin{aligned}
& T=\left\{A \in \mathrm{WFF}: v_{0}(A)=1\right\}, \\
& F=\left\{A \in \mathrm{WFF}: v_{0}(A)=0\right\} .
\end{aligned}
$$

For each $v \neq v_{0}$, either $v(B)=0$ for some $B \in T$ or $v(B)=1$ for some $B \in F$. Thus, $v$ confirms the sequent $T \vdash F$. It follows that the sequent is $V$-valid (since it is confirmed by every valuation except $\left.v_{0}\right)$. Since $v_{0}$ does not confirm the argument, however, $v_{0}$ is not $\mathbb{L}^{M}(V)$-consistent. In other words, $v_{0} \notin \mathbb{V}\left(\mathbb{L}^{M}(V)\right)$.

Thus, we know that the multiple conclusion framework offers categoricity with respect to the same valuational spaces as the signed framework. This is not altogether surprising. There is indeed formal evidence that the two frameworks are mere notational variations of each other. Although Smiley did not show it, it seems likely that he had in mind a translation between a signed and a nonsigned sequent which preserves both validity and categoricity.

In fact, it is straightforward to define a faithful translation between signed sequents and multiple conclusion sequents. First, let $A_{1}, \ldots, A_{n}, \ldots, \vdash B_{1}, \ldots, B_{m}, \ldots$ be a multiple conclusion sequent. Its translation $\tau\left(A_{1}, \ldots, A_{n}, \ldots, \vdash B_{1}, \ldots, B_{m}, \ldots\right)$ is the signed sequent $+A_{1}, \ldots,+A_{n},-B_{2}, \ldots,-B_{m}, \ldots, \vdash+B_{1}$. We can then prove that every multiple conclusion sequent $\Gamma \vdash \Delta$ is satisfied by valuation $v$ just in case $\tau(\Gamma \vdash \Delta)$ is satisfied by $v$. For left-to-right, assume that $\tau(\Gamma \vdash \Delta)$ is not satisfied by $v$. Then $+B_{1}$ is incorrect on $v$ and each of $+A_{1}, \ldots,+A_{n},-B_{2}, \ldots,-B_{m}, \ldots$, is correct on $v$. Thus, for each $i, v\left(B_{i}\right)=0$ and $v\left(A_{i}\right)=1$, as required. The other direction is equally straightforward.

Correspondingly, for a signed sequent $+A_{1}, \ldots,+A_{n}, \ldots,-B_{1}, \ldots,-B_{m}$, $\ldots, \vdash \pm C$, the translation is

$$
\begin{aligned}
\tau & \left(+A_{1}, \ldots,+A_{n}, \ldots,-B_{1}, \ldots,-B_{m}, \ldots, \vdash \pm C\right) \\
& = \begin{cases}A_{1}, \ldots, A_{n}, \ldots, \vdash C, B_{1}, \ldots, B_{m}, \ldots, & \text { if }+C, \\
C, A_{1}, \ldots, A_{n}, \ldots, \vdash B_{1}, \ldots, B_{m}, \ldots, & \text { if }-C .\end{cases}
\end{aligned}
$$

Again, the translation is faithful in the sense that a valuation $v$ satisfies an inference $\Gamma^{*} \vdash A^{*}$ if and only if it satisfies $\tau\left(\Gamma^{*} \vdash A^{*}\right)$.

There is no doubt then that the two frameworks are intimately connected. But for all the technical similarities, it might be worth investigating whether the two frameworks have different philosophical merits:

The close formal relationship between bilateral calculi and their multipleconclusion cousins, however, should not blind us to what is, for present purposes, a crucial philosophical difference. (Rumfitt $[28$, p. 810]) 
What is the crucial difference? Rumfitt is not at all impressed by the capacities of the multiple conclusion framework. He argues at length against multiple conclusion in [31] and also broaches the topic when he presents his bilateralism. Rumfitt [28, pp. 795-96] claims that "not only is it doubtful whether people actually give such [multiple conclusion] arguments, it is also doubtful whether we can attain any intelligible conception of them." Rumfitt suggests that we understand multiple conclusion as a "meta-logical remark" of the form "if these propositions are true, then some other propositions cannot all be false." Such a remark, he continues, is a poor representation of inference, since the involved propositions are merely mentioned.

Rumfitt's position, that multiple conclusion frameworks are somehow too artificial to represent actual inference, is shared by several others in the inferentialist literature. For example, according to Dummett, ${ }^{16}$

\begin{abstract}
Sequents with two or more sentences in the succedent, by contrast, have no straightforwardly intelligible meaning, explicable without recourse to any logical constant. Asserting $\mathbf{A}$ and asserting $\mathbf{B}$ is tantamount to asserting $\ulcorner\mathbf{A}$ and $\mathbf{B}\urcorner$; so, although the sentences in the antecedent of a sequent are in a sense conjunctively connected, we can understand the significance of a sequent with more than one sentence in the antecedent without having to know the meaning of 'and'. But, in a succedent comprising more than one sentence, the sentences are connected disjunctively; and it is not possible to grasp the sense of such a connection otherwise than by learning the meaning of the constant 'or'. [6, p. 187]
\end{abstract}

Rumfitt's objection appears to be inspired by Dummett's analysis. Oddly enough, however, Dummett's analysis relies precisely on a reduction of denial to assertion of negation - the very strategy that Rumfitt has set out to reject. Dummett is correct in claiming that conjunction and disjunction are asymmetric with respect to assertion. However, this does not show that conjunctive commas are conceptually kosher prior to their object language counterparts, whereas disjunctive commas are not. Unlike the antecedent-side commas, the disjunctive (right-hand) commas ought not to be treated assertorically. Rather, we will think of the normative constraint imposed by a multiple conclusion consequence relation as saying that one should not (on pain of incoherence) assert all premises and deny all conclusions simultaneously. With such a reading there is not necessarily a genuine disjunctive element: $\langle\Gamma, \Delta\rangle$ is invalid just in case we assert $A_{1}$, and we assert $A_{2}$, and so on, for each $A_{i} \in \Gamma$, and we deny $B_{1}$, and we deny $B_{2}$, and so on, for each $B_{i} \in \Delta$. Summed up, to the extent that it is a worry at all that there are metalevel conceptions of connectives, Dummett seems to have put too much emphasis on the role played by the interaction between assertion and conjunction. ${ }^{17}$

\title{
5 Beyond Assertion and Denial
}

This counter to Dummett can be made more precise using a framework offered by Restall [24], [25]). Restall says:

We can think of the rules for the connectives as giving instructions on how to treat assertions and denials—at least with regard to whether or not these assertions and denials are out of bounds. [25, p. 245]

On Restall's approach we consider structures deceptively similar to arguments, that is, pairs of collections of statements, written $[\Gamma: \Delta]$. Call such a structure a position. We say further that for a position $[\Gamma: \Delta], \Gamma$ is the set of asserted propositions and 
$\Delta$ is the set of denied propositions. A position $[\Gamma: \Delta]$ such that $\Gamma \cap \Delta \neq \emptyset$ is an incoherent position (i.e., positions where the very same proposition is both asserted and denied). For an incoherent position $[\Gamma: \Delta]$, we write $\Gamma \vdash \Delta .{ }^{18}$

Formally, there is a lot of freedom with respect to how the sequent framework fixes the associated notion of incoherence. We can, for example, strengthen the notion of incoherence by adding structural constraints on the sequent calculus. Restall suggests that if $[\Gamma: \Delta]$ is coherent, and $\Gamma^{\prime} \subseteq \Gamma$ and $\Delta^{\prime} \subseteq \Delta$, then $\left[\Gamma^{\prime}: \Delta^{\prime}\right]$ is also coherent (weakening); if $[\Gamma: \Delta]$ is coherent, then so is either $[\Gamma, \mathrm{A}: \Delta]$ or $[\Gamma: \Delta, \mathrm{A}]$ (transitivity). ${ }^{19}$

More interesting for our purposes are the position rules for logical connectives. We will consider the position rules for a logical connective to be conceptually antecedent to its truth conditions. Let us take conjunction as an example. If $[\Gamma: \Delta, \mathrm{A} \wedge \mathrm{B}]$ is coherent, then either $[\Gamma: \Delta, \mathrm{A}]$ is coherent or $[\Gamma: \Delta, \mathrm{B}]$ is coherent (or both). As with the structural constraints above, we recognize sequent rules by taking the contraposition of the constraints. In other words, if both $[\Gamma: \Delta, \mathrm{A}]$ and $[\Gamma: \Delta, \mathrm{B}]$ are incoherent (i.e., $\Gamma \vdash \Delta$, $\mathrm{A}$ and $\Gamma \vdash \Delta$, B), then $[\Gamma: \Delta, \mathrm{A} \wedge \mathrm{B}]$ is incoherent (i.e., $\Gamma \vdash \Delta$, $\mathrm{A} \wedge \mathrm{B}$ ). Put more informally, if we assert $\mathrm{A}$ and we assert $\mathrm{B}$, then we ought to assert $\mathrm{A} \wedge \mathrm{B}$. Accordingly, we might want to express that if you deny either $\mathrm{A}$ or $\mathrm{B}$, you ought to deny $\mathrm{A} \wedge \mathrm{B}$. This leads to the following rule: if $[\Gamma, \mathrm{A}, \mathrm{B}: \Delta]$ is incoherent, then $[\Gamma, \mathrm{A} \wedge \mathrm{B}: \Delta]$ is incoherent. Again, these are simply notational variations of sequent rules for conjunction:

$$
\frac{\Gamma, A, B \Rightarrow \Delta}{\Gamma, A \wedge B \Rightarrow \Delta} \quad \frac{\Gamma \Rightarrow A, \Delta \quad \Gamma \Rightarrow B, \Delta}{\Gamma \Rightarrow A \wedge B, \Delta}
$$

On the position interpretation, multiple conclusion is not a mere "metalogical remark": there is so far no talk about truth conditions at all but only about assertion and denial. There are two advantages to this. First, we are free to explore ways in which the truth-conditional semantics of logical connectives are "read off" the pragmatic rules governing positions. Unsurprisingly, this will be a question of categoricity. More contentiously, one might think of the rules governing the positions as constitutive for the sense of logical connectives. Thus paraphrased, the question of whether or not these very rules also determine the reference is precisely the question of categoricity.

Second, we need not stop with assertion and denial. There is no tertium non datur for speech acts. We might think that there is more than one type of denial, just as we sometimes think that there is more than one type of negation. Perhaps, for example, there is a type of denial which corresponds roughly to metalinguistic negation. For example, in cases of presupposition failure we can deny propositions like Sue stopped smoking without denying its content. ${ }^{20}$ Or, alternatively, there might be an inferentially significant speech act which falls, in some sense, between assertion and denial. For instance, propositions with both vague expressions and paradoxical propositions are sometimes considered neither true nor false and thus perhaps neither assertible nor deniable. A middle speech act might correspond to doubting, being agnostic, or withholding assent/dissent. ${ }^{21}$ Paraphrasing Rumfitt's informal reading of,+- as "A? Yes" and "A? No," we may for example introduce a reading of a third position as "A? Dunno." 22 Positions are not two-party systems: Everyone's invited. ${ }^{23}$ 


\section{Multilateralism and Many-Valued Logics}

With the position interpretation of sequent calculus in mind, I will move on to consider an expansion of the bilateralist project. The above section should serve to motivate the thought that once we appreciate the advantages of going bilateral, there is nothing stopping us from expanding to multilateralism for logical connectives.

Bilateral rules give us categoricity for valuation spaces in the Boolean universe. Multilateralism will give us categoricity for arbitrary finite many-valued matrices. The idea is twofold. Formally we introduce sequents which are $n$-sided and show how we can give a categoricity result for many-valued logics. Informally, we allow inference rules to operate on speech acts other than assertion and denial. Pursuing the inferentialist thought, we then say that the truth conditions of logical connectives are carved out by these more fine-grained rules.

One way of thinking about the generalization is to say that the logic now comes with $n$-signed formulas, each index indicating a distinct speech act. But, with sequent calculus, we simply indicate the signs with locations in $n$-sided positions. Unsurprisingly, bilateral systems will simply be the special case where the positions are two-sided. In this sense, the classical bilateral system is just the standard sequent calculus for classical logic.

What we need is a proof-theoretic framework that generalizes the bilateral systems, and which ultimately leads to the required categoricity result. Fortunately, such a framework is already available in the form of $n$-sided sequent calculus, pioneered in Schröter [33] and Rousseau [26] and subsequently developed further in Baaz, Fermüller, and Zach [1]. ${ }^{24}$

We simply start out with a standard sequent $\Gamma_{0} \Rightarrow \Gamma_{1}$ and then turn it into a position-like structure:

$$
\Gamma_{0} \mid \Gamma_{1}
$$

We read this two-sided sequent disjunctively as saying, informally, that either something in $\Gamma_{0}$ is denied or something in $\Gamma_{1}$ is asserted. In other words, the informal gloss is along the following lines:

$$
\left(\gamma_{0}^{1} \vee \gamma_{0}^{2} \vee \cdots \vee \gamma_{0}^{n}\right) \vee\left(\gamma_{1}^{1} \vee \gamma_{1}^{2} \vee \cdots \vee \gamma_{1}^{n}\right),
$$

where each $\gamma_{0}^{i} \in \Gamma_{0}$ is denied, and each $\gamma_{1}^{i} \in \Gamma_{1}$ is asserted. $^{25}$

Recall the general Definition 2.1 of valuations, valuation spaces, and the universe of valuations. In a finite many-valued semantics, there will be three or more values in the set of truth values $\mu_{1}, \ldots, \mu_{n} \in \mathcal{V}$, and one or more of these will be designated.

Definition 6.1 ( $n$-sided sequents) Let an $n$-sided sequent be an ordered $n$-tuple $\left\langle\Gamma_{1}, \ldots, \Gamma_{n}\right\rangle$ where each $\Gamma_{i}(1 \leq i \leq n)$ is a (possibly empty) set of formulas (of some set of $W F F \mathrm{~s}$ ). Let $\mu_{1}, \ldots, \mu_{n}$ be the truth values of the many-valued semantics. A valuation $v$ confirms an $n$-sided sequent $\left\langle\Gamma_{1}, \ldots, \Gamma_{n}\right\rangle$ just in case, for some $\Gamma_{i}$, there is a formula $A \in \Gamma_{i}$ such that $v(A)=\mu_{i}$. Otherwise, $v$ falsifies the sequent.

Definition 6.2 ( $V$-validity) Let $V$ be a valuation space. An $n$-sided sequent $\left\langle\Gamma_{1}, \ldots, \Gamma_{n}\right\rangle$ is $V$-valid just in case it is confirmed by every $v \in V$.

Instead of the usual turnstile notation we will use vertical bars to separate positions in an $n$-sided sequent:

$$
\Gamma_{1}|\ldots| \Gamma_{n}
$$


Inference rules operating on $n$-sided sequents are pairs consisting of a set of $n$-sided sequents (the premises) and an $n$-sided sequent (the conclusion):

$$
\frac{\left(\Gamma_{1}^{1}|\ldots| \Gamma_{n}^{1}\right) \ldots\left(\Gamma_{1}^{m}|\ldots| \Gamma_{n}^{m}\right)}{\Gamma_{1}|\ldots| \Gamma_{n}} .
$$

Importantly, the sequent premises are conjunctive (as in standard sequent calculus), so that the combination of all the premises are in conjunctive normal form. We call the proof-theoretic framework consisting of inference rules of this type $\{$ nMSEQ $\}$-nMSEQ. ${ }^{26}$

Definition 6.3 Let an ( $n$-sided sequent) logic $L^{n}$ be a set of \{nMSEQ\}-nMSEQ inferences (with respect to some language and set of $W F F \mathrm{~s}$ ). We say that an inference is $L^{n}$-valid if it is in $L^{n}$.

Definition 6.4 Let $V$ be a valuation space, and let $L^{n}$ be a logic:

$$
\mathbb{L}^{n}(V)={ }_{d f}\left\{\{\text { nMSEQ }\} \text {-nMSEQ } \in L^{n}:\{\text { MSEQ }\} \text {-MSEQ is } V \text {-valid }\right\} .
$$

With the \{nMSEQ\}-nMSEQ framework we can complete a project started in [27]. Rumfitt extends the bilateral system to give a categoricity result for strong Kleene, K3. ${ }^{27}$ With a multilateral approach, however, we can get categoricity for any finite many-valued logic. In addition, we can treat logics with preservation properties that involve more than one value.

Theorem 6.5 (\{nMSEQ\}-nMSEQ) For any valuation space $V \subseteq U, V=$ $\mathbb{V}\left(\mathbb{L}^{n}(V)\right)$.

Proof Suppose that $v_{0} \notin V$. We show that $v_{0} \notin \mathbb{V}\left(\mathbb{L}^{n}(V)\right)$. Define the following sets:

$$
\begin{gathered}
\Gamma_{1}=\left\{A \in \mathrm{WFF}: v_{0}(A) \neq \mu_{1}\right\}, \\
\Gamma_{2}=\left\{A \in \mathrm{WFF}: v_{0}(A) \neq \mu_{2}\right\}, \\
\vdots \\
\Gamma_{n}=\left\{A \in \mathrm{WFF}: v_{0}(A) \neq \mu_{n}\right\} .
\end{gathered}
$$

For each $v \neq v_{0}, v$ confirms the $n$-sided sequent $\Gamma_{1}\left|\Gamma_{2}\right| \cdots \mid \Gamma_{n}$. For, since $v \neq v_{0}$, there is a formula $A$ such that $v(A) \neq v_{0}(A)$. Assume that $v_{0}(A)=\mu_{i}$. Then for some $j \neq i, v(A)=\mu_{j}$, and since, by definition, $A \in \Gamma_{k}$, for every $\Gamma_{k}$ where $k \neq i$, we know that $A \in \Gamma_{j}$ in particular. Thus, $v$ confirms $\Gamma_{1}\left|\Gamma_{2}\right| \cdots \mid \Gamma_{n}$, as required. On the other hand, $v_{0}$ does not confirm $\Gamma_{1}\left|\Gamma_{2}\right| \cdots \mid \Gamma_{n}$. For, if it did, then for some $i$, there is a formula $A \in \Gamma_{i}$ such that $v_{0}(A)=\mu_{i}$. But, by definition, if $A \in \Gamma_{i}$, then $v_{0}(A)=\mu_{i}$.

Thus $\Gamma_{1}\left|\Gamma_{2}\right| \cdots \mid \Gamma_{n}$ is $V$-valid (every $v$ except $v_{0}$ confirms it), and since $v_{0}$ does not confirm it, we have $v_{0} \notin \mathbb{V}\left(\mathbb{L}^{n}(V)\right)$.

The result tells us little, however, about the proof-theoretic specifics. In the $\{$ MSEQ\}-MSEQ and $\{$ SEQ $*$ \}-SEQ* frameworks, we had corresponding formalizations of systems for classical logic. But the above theorem gives us no particulars about proof systems. In fact, it is a key aspect of the refutation sequents that they might involve infinite sets $\Gamma_{i}$. Recall that the refutation sequent is defined over a valuation and thus involves every formula in the language. But, if one is interested in PTS, it is a natural constraint that the inference rules-and thus the sequents-be 
finitary. In particular, if we think of sequent rules as an agent's rational management of assertions, denials, and other speech acts, then one might want to keep each location in the sequent finite.

Yet, for all that has been said so far there is no guarantee that inference rules of finite $n$-sequents have the requisite expressive power. Perhaps some such systems suffer from noncategoricity. Fortunately, that is not the case. We can give finite $n$-sided sequent rules that capture precisely the truth conditions of a logical connective. The idea is roughly as follows: For each connective, and each location in the sequent, there is a single rule which states the necessary and sufficient grounds for introducing the connective in the location. Even more tentatively, we can think of these introduction rules as stating the respective conditions under which one ought to assert or deny or doubt, say, $A \vee B$.

Let us look to an example. Consider K3 mentioned above and in particular the truth table for its disjunction:

\begin{tabular}{|c|ccc|}
\hline$f_{\vee}$ & 1 & $\mathrm{i}$ & 0 \\
\hline 1 & 1 & 1 & 1 \\
$\mathrm{i}$ & 1 & $\mathrm{i}$ & $\mathrm{i}$ \\
0 & 1 & $\mathrm{i}$ & 0 \\
\hline
\end{tabular}

The K3-matrix has three values, of which only 1 is designated. Consider the following three 3 -sided sequent rules where each $\Gamma_{i}$ is finite:

$\frac{\left(\Gamma_{0}, A\left|\Gamma_{i}\right| \Gamma_{1}\right)}{\left.\Gamma_{0}, A \vee B\left|\Gamma_{0}, B\right| \Gamma_{i} \mid \Gamma_{1}\right)} \frac{\Gamma_{0}\left|\Gamma_{i}\right| \Gamma_{1}, A, B}{\Gamma_{0}\left|\Gamma_{i}\right| \Gamma_{1}, A \vee B}$
$\frac{\left(\Gamma_{0}\left|\Gamma_{i}, A, B\right| \Gamma_{1}\right)\left(\Gamma_{0}, A\left|\Gamma_{i}, A\right| \Gamma_{1}\right)}{\left(\Gamma_{0}, B\left|\Gamma_{i}, B\right| \Gamma_{1}\right)}$
$\Gamma_{0}\left|\Gamma_{i}, A \vee B\right| \Gamma_{1}$

How do we interpret the above $n$-sided sequent rules? For simplicity, simply let $\Gamma_{0}=\Gamma_{i}=\Gamma_{1}=\varnothing$. The top leftmost rule and the top rightmost rule give the 0 -conditions and 1-conditions for disjunction in $\mathbf{K 3}$, respectively. Corresponding to the position-interpretation of standard two-sided sequents we are invited to interpret these proof conditions as denial conditions and assertion conditions, respectively. A disjunction is deniable just in case each of its disjuncts are; a disjunction is assertible just in case at least one of its disjuncts are.

In fact, given that the $\mathbf{K} \mathbf{3}$ matrices are simply Boolean (or classical) in the absence of the third value $i$, the assertion and denial conditions specified by these rules are identical to those of both Restall's classical sequent system and Rumfitt's signed natural deduction system. We can think of this as classical recapture in the limit cases, that is, in the leftmost and rightmost locations in the sequents. ${ }^{28}$

Let us move on to the bottom rule. It gives the $i$-conditions for a disjunction. As is evident from the truth table above, the K3-disjunction has the value $i$ just in case either both disjuncts also have the value $i$, or one disjunct has value $i$ and the other 0 . However, as we saw above, the sequent rule expresses this in conjunctive normal form, rather than disjunctive normal form. That is, each premise-sequent is a disjunction, whereas the premises themselves are combined as conjunctions. Thus the sequent premises combined say that three conditions are necessary and sufficient for the disjunction having value $i: A$ or $B$ takes value $i ; A$ takes value 0 or value $i$; and $B$ takes value 0 or value $i$. 
Is there a speech act, or a propositional attitude, that corresponds to the value $i$ in the sense that assertion corresponds to the value 1? Let us try on one of the suggestions from Section 5 and interpret $i$-conditions as conditions for doubting (or being agnostic about) a proposition. The philosophical motivation is that $\mathbf{K 3}$ accommodates indeterminate or "gappy" propositions, and it seems plausible that such propositions can neither be correctly asserted nor denied. ${ }^{29}$

Let us quickly also look at the $\mathbf{K} \mathbf{3}$ negation, which is determined by the following rules:

$$
\frac{\Gamma_{0}\left|\Gamma_{i}\right| \Gamma_{1}, A}{\Gamma_{0}, \neg A\left|\Gamma_{i}\right| \Gamma_{1}}, \quad \frac{\Gamma_{0}\left|\Gamma_{i}, A\right| \Gamma_{1}}{\Gamma_{0}\left|\Gamma_{i}, \neg A\right| \Gamma_{1}}, \quad \frac{\Gamma_{0}, A\left|\Gamma_{i}\right| \Gamma_{1}}{\Gamma_{0}\left|\Gamma_{i}\right| \Gamma_{1}, \neg A}
$$

This imposes a constraint on a valuation space $V$ as follows: For every valuation $v \in V$,

$$
v(\neg A)= \begin{cases}1 & \text { if } v(A)=0, \\ \mathrm{i} & \text { if } v(A)=i, \\ 0 & \text { if } v(A)=1 .\end{cases}
$$

It should be clear from this that $n$-sided sequents can be used to give categorical axiomatization of a range of logics. That is not to say, however, that any set of $n$-sided sequent rules will do the trick. What we know from the above is that for any finite many-valued logic, there is a categorical axiomatization in the \{nMSEQ\}-nMSEQ framework. It is nonetheless straightforward to see that there are systems that will lead to both overdetermination and underdetermination. For example, for a connective $\oplus$ we might have the following rules:

$$
\begin{gathered}
\frac{\left(\Gamma_{0}, A\left|\Gamma_{i}\right| \Gamma_{1}\right)\left(\Gamma_{0}, B\left|\Gamma_{i}\right| \Gamma_{1}\right)}{\Gamma_{0}, A \oplus B\left|\Gamma_{i}\right| \Gamma_{1}}, \frac{\left(\Gamma_{0}\left|\Gamma_{i}\right| \Gamma_{1}, A\right) \quad\left(\Gamma_{0}\left|\Gamma_{i}\right| \Gamma_{1}, B\right)}{\Gamma_{0}\left|\Gamma_{i}\right| \Gamma_{1}, A \oplus B}, \frac{\Gamma_{0}\left|\Gamma_{i}, A, B\right| \Gamma_{1}}{\Gamma_{0}\left|\Gamma_{i}, A \oplus B\right| \Gamma_{1}} .
\end{gathered}
$$

The constraints imposed by the above inference rules for $\oplus$ yield the following incomplete truth table:

\begin{tabular}{|c|ccc|}
\hline$f_{\oplus}$ & 1 & $\mathrm{i}$ & 0 \\
\hline 1 & 1 & $\mathrm{i}$ & $?$ \\
$\mathrm{i}$ & $\mathrm{i}$ & $\mathrm{i}$ & $\mathrm{i}$ \\
0 & $?$ & $\mathrm{i}$ & 0 \\
\hline
\end{tabular}

Similarly, we might have a situation where a set of inference rules are in conflict. In fact, this is a situation familiar from the inference rules for tonk. These rules offer more than one way of determining the truth table. Recall the standard sequent rules for tonk:

$$
\frac{\Gamma_{0}, B \mid \Gamma_{1}}{\Gamma_{0}, A \operatorname{tonk} B \mid \Gamma_{1}}, \quad \frac{\Gamma_{0} \mid A, \Gamma_{1}}{\Gamma_{0} \mid A \operatorname{tonk} B, \Gamma_{1}} .
$$

In terms of (Boolean) truth conditions, the above rules for tonk both overdetermine and underdetermine. First, the rules are in conflict with respect to what happens when $B$ is in the 0 -location and $A$ in the 1-location. Second, the rules are silent about what happens when $B$ is in the 1-location and $A$ in the 0-location. Essentially, the overdetermination witnessed here is a precise formulation of the worry voiced 
by Stevenson and Wagner about tonk. What they failed to realize is that the phenomenon is general and can thus be studied independently of tonk and the Boolean setting.

\section{Conclusion}

Categoricity is a formal approach to the question of how inference rules determine the meaning of logical connectives. It captures formally the connection between proof conditions and truth conditions. The present investigation shows how sensitive this approach is to the details of proof-theoretic frameworks. With the help of $n$-sided sequents we have proved categoricity for arbitrary finite many-valued logics, and thus taken proof-theoretic semantics beyond its traditional revisionistic boundaries. The notion of categoricity also offers a diagnosis of rogue connectives like tonk that brings together both proof conditions and truth conditions. Finally, by extending bilateralism to a multilateralist account of logical connectives, we provide an informal reading of $n$-sided sequents in terms of the norms governing speech acts.

\section{Notes}

1. As a reminder, tonk has the following intro- and elim-rules:

$$
\frac{A}{A \text { tonk } B}{ }^{(i n t r o)}, \quad \frac{A \text { tonk } B}{B} \text { (elim) }
$$

Assuming transitivity, tonk gives a provability relation where anything is provable from a nonempty set of premises. In the presence of most negations, it proves anything from any set of premises.

2. See, for example, Milne [19], Read [23], Schroeder-Heister [32], and Tennant [39] for some proposals.

3. This is not the place for details, but the arguments typically cite the fact that classical negation is nonconservative over the (intuitionistic) $\rightarrow$-fragment in standard natural deduction systems or that the negation rules do not have the standard detour conversions applied in the normalization theorem.

4. The admissible valuations for classical logic are the ones that respect the standard truth functions associated with the classical connectives. Let an assignment be a function $v_{0}$ from propositional variables to truth values. Then an admissible valuation is a valuation recursively defined over the truth functions from an assignment. It is straightforward that for each assignment there is a unique valuation.

5. The degenerate instances where no formulas are discharged in the subderivations will not help. The valuation $v_{@}$ defined below in note 7 is one example of why these disjunction rules cannot work to determine the truth table.

6. In fact, the classical reductio rule, RED, is sufficient alone, since the vacuous case where we do not discharge any assumptions is simply EFQ. In sequent style natural deduction, we can see this by the following derivation, applying the structural rule weakening $(K)$ :

$$
\frac{\frac{\Gamma \vdash B}{\Gamma, \neg A \vdash B}(K) \frac{\Gamma \vdash \neg B}{\Gamma, \neg A \vdash \neg B}}{\Gamma \vdash A} \text { (RED) }
$$


7. Importantly, $v^{*}$ is not the only nonstandard valuation that cannot be dealt with in $\{\mathrm{SEQ}\}$-SEQ. Take, for instance, the valuation $v^{@}$ such that $v^{@}(A)=0$ just in case $\nvdash A$. I owe this example to Elia Zardini.

8. The notation here is adapted from Hardegree [14]. See also Dunn and Hardegree [8] for further details.

9. In Shoesmith and Smiley [34] the notion of categoricity is different from what is presented here. Instead of valuations, they consider partitions of formulas into designated and nondesignated. Since a valuation, regardless of the truth values, will determine a partition but not necessarily vice versa, our present definition is more fine-grained.

10. Categoricity also plays an implicit role in questions of logicality (see especially Bonnay $[3])$.

11. An alternative approach that will not be pursued here is to insist that the universe of valuations be limited to valuations where for some $A \in \mathrm{WFF}, v(A)=0$. This is the basis of closely related research in Belnap and Massey [2] and Garson [11], [12]. Although their findings are very interesting, the assumption that each valuation takes something to false is undesirable for present purposes. Denis Bonnay has also suggested that it might be worth restricting the scope to compositional valuations. Although this would rule out nonintended valuations such as $v^{*}$, I would prefer the compositionality of the semantics to follow proof-theoretically.

12. For the debate following Rumfitt's paper, see Dummett [7], Gibbard [13], Rumfitt [29], Ferreira [9], and Rumfitt [30].

13. More broadly one could interpret the signs as the propositional attitudes of acceptance and rejection rather than speech acts.

14. Standard natural deduction systems for classical logic fail on this account because some theorems (e.g., Peirce's law) in the $\rightarrow$-fragment of the language are only provable using inference rules for the classical negation.

15. There is a variety of proof systems for classical logic, which is based on multiple conclusions. See for example Boričić [4] and Ungar [41] for Prawitz-style natural deduction system for classical logic. However, a sequent calculus presentation is more typical (see, e.g., Troelstra and Schwichtenberg [40]).

16. Tennant $[38$, p. 320] is another: "In normal practice, arguments take one from premises to a single conclusion."

17. Interestingly, we can dualize and say, equivalently, that the argument $\langle\Gamma, \Delta\rangle$ is valid just in case we deny $A_{1}$, or we deny $A_{2}$, and so on, $\mathrm{OR}$, we assert $B_{1}$ or assert $B_{2}$. This perspective will become useful in what follows.

18. I have deviated somewhat from Restall's preferred characterization of positions. He uses an equivalent reading of sequents where the antecedent set $\Gamma$ are the denied propositions, and the succedent set $\Delta$ are the asserted propositions. I owe this observation to a referee. 
19. One might have conceptions of assertion and denial (and associated conceptions of coherence) which drop any or all of these constraints. The difference, as Restall remarks, amounts roughly to a variety of substructural sequent calculi. Additionally, the framework builds in contraction by working with sets rather than multisets. However, we will not pursue substructural issues any further in what follows.

20. A similar consideration is made in Rumfitt [27, pp. 226-27] as internal versus external rejection.

21. What is expressed by saying that one doubts something might vary. If I say "There is mate in two" and you reply "I doubt it," a plausible reading is that you are denying what I said. What is of interest for the present purposes is the weaker attitude where one will neither assert nor deny.

22. See [7] for a discussion of "A? Can't say."

23. A final advantage to the position interpretation is that it avoids a problem with RumfittSmiley-style bilateral systems. Recall that these systems have rules that operate on signed formulas. Informally, the rules govern the connections between assertion and denial of propositions with logical connectives. However, in the coordination principles, the signed formulas occur as assumptions that are discharged by the application of the rule. This is misleading. What is being assumed for the sake of argument (the hypothetical) is neither asserted nor denied. In fact, what this reveals is that in unilateral systems, it would be wrong to think of inferences as operating exclusively on asserted propositions. There are two modes: asserted propositions and assumed propositions. Thus, explicating assertions and denials with signs,,+- , in assumption position confuses matters. What is being assumed is a proposition, not a speech act. Propositionalizing by saying that we assume that $A$ is denied/asserted would sabotage the signs. For, if the signs are propositionalized in assumption position, why are they nonembeddable? Importantly, there is no corresponding problem with sequent calculi under the position interpretation.

24. $n$-sided systems are also used to give proof systems with more than one derivability relation in Hjortland [15].

25. Feel free to think of the subscripts as signs, but in the actual sequents the location determines the judgment type.

26. An $n$-sided sequent calculus has corresponding structural rules; for example, weakening, for a 3-valued Łukasiewicz logic:

$$
\frac{\Gamma_{1}\left|\Gamma_{2}\right| \Gamma_{3}}{\Gamma_{1}, A\left|\Gamma_{2}\right| \Gamma_{3}}\left(K_{1}\right), \quad \frac{\Gamma_{1}\left|\Gamma_{2}\right| \Gamma_{3}}{\Gamma_{1}\left|\Gamma_{2}, A\right| \Gamma_{3}}\left(K_{2}\right), \quad \frac{\Gamma_{1}\left|\Gamma_{2}\right| \Gamma_{3}}{\Gamma_{1}\left|\Gamma_{2}\right| \Gamma_{3}, A}\left(K_{3}\right)
$$

For further details, including cut elimination, soundness, and completeness for $n$-sided calculi, see for example [1] and references therein.

27. See Priest [21, pp. 22-24] for details.

28. Notice, however, that this is a special feature of $\mathbf{K 3}$, and need not hold in other manyvalued matrices we consider. We are free to insist that the presence of a non-Boolean value impacts on assertion and denial as well. 
29. Think, for example, of the way in which $\mathbf{K} \mathbf{3}$ is applied to ungrounded propositions in the literature on semantic paradoxes (see, e.g., Kripke [17] and Field [10]).

\section{References}

[1] Baaz, M., C. Fermüller, and R. Zach, "Systematic construction of natural deduction systems for many-valued logics," pp. 208-13 in Proceedings of the 23rd International Symposium on Multiple-Valued Logic, IEEE Press, New York, 1993. 458, 464

[2] Belnap, N. D., Jr., and G. J. Massey, "Semantic holism," Studia Logica, vol. 49 (1990), pp. 67-82. Zbl 0706.03005. MR 1078439. DOI 10.1007/BF00401554. 463

[3] Bonnay, D., and D. Westerståhl, "Logical consequence inside out," pp. 193-202 in Logic, Language and Meaning, Springer, Berlin, 2010. 463

[4] Boričić, B. R., "On sequence-conclusion natural deduction systems," Journal of Philosophical Logic, vol. 14 (1985), pp. 359-77. MR 0816241. DOI 10.1007/BF00649481. 463

[5] Carnap, R., Formalization of Logic, Harvard Univ. Press, Cambridge, Mass., 1943. MR 0007892. 447

[6] Dummett, M., The Logical Basis of Metaphysics, Harvard Univ. Press, Cambridge, Mass., 1991. 446, 456

[7] Dummett, M., “'Yes,' 'no' and 'can't say,”' Mind, vol. 111 (2002), pp. 289-95. MR 2133868. DOI 10.1093/mind/111.442.289. 463, 464

[8] Dunn, J. M., and G. M. Hardegree, Algebraic Methods in Philosophical Logic, vol. 41 of Oxford Logic Guides, Oxford Univ. Press, New York, 2001. Zbl 1014.03002. MR 1858927. 455, 463

[9] Ferreira, F., "The co-ordination principles: A problem for bilateralism," Mind, vol. 117 (2008), pp. 1051-57. MR 2804680. DOI 10.1093/mind/fzn036. 463

[10] Field, H., Saving Truth from Paradox, Oxford Univ. Press, Oxford, 2008. MR 2723032. DOI 10.1093/acprof:oso/9780199230747.001.0001. 465

[11] Garson, J. W., "Natural semantics: Why natural deduction is intuitionistic," Theoria (Stockholm), vol. 67 (2001), pp. 114-39. MR 1885532. DOI 10.1111/j.1755-2567.2001.tb00200.x. 463

[12] Garson, J. W., "Expressive power and incompleteness of propositional logics," Journal of Philosophical Logic, vol. 39 (2010), pp. 159-71. Zbl 1193.03017. MR 2608137. DOI 10.1007/s10992-009-9120-z. 463

[13] Gibbard, P., "Price and Rumfitt on rejective negation and classical logic," Mind, vol. 111 (2002), pp. 297-303. MR 2133869. DOI 10.1093/mind/111.442.297. 463

[14] Hardegree, G. M., "Completeness and super-valuations," Journal of Philosophical Logic, vol. 34 (2005), pp. 81-95. Zbl 1085.03008. MR 2138856. DOI 10.1007/s10992-004-6302-6. 463

[15] Hjortland, O. T., "Logical pluralism, meaning-variance, and verbal disputes," Australasian Journal of Philosophy, vol. 91 (2013), pp. 355-73. 464

[16] Hodes, H., "On the sense and reference of a logical constant: The foundations of mathematics and logic," The Philosophical Quarterly, vol. 54 (2004), pp. 134-65. MR 2050642. DOI 10.1111/j.0031-8094.2004.00345.x. 446

[17] Kripke, S., "Outline of a theory of truth," Journal of Philosophy, vol. 72 (1975), pp. 690-716. 465

[18] Milne, P., "Classical harmony: Rules of inference and the meaning of the logical constants," Synthese, vol. 100 (1994), pp. 49-94. Zbl 0810.03002. MR 1308252. DOI 10.1007/BF01063921. 446

[19] Milne, P., "Harmony, purity, simplicity and a 'seemingly magical fact,"' The Monist, vol. 85 (2002), pp. 498-534. 446, 462 
[20] Prawitz, D., "Ideas and results in proof theory," pp. 235-307 in Proceedings of the 2nd Scandinavian Logic Symposium (Oslo, 1970), edited by J. Fenstad, vol. 63 of Studies in Logic and the Foundations of Mathematics, North-Holland, Amsterdam, 1971. MR 0387024. 446

[21] Priest, G., An Introduction to Non-classical Logic: From If to Is, 2nd ed., Cambridge Univ. Press, Cambridge, 2008. MR 2412933. DOI 10.1017/CBO9780511801174. 464

[22] Prior, A. N., "The runabout inference ticket," Analysis, vol. 21 (1961), pp. 38-39. 446

[23] Read, S., "Harmony and autonomy in classical logic," Journal of Philosophical Logic, vol. 29 (2000), pp. 123-54. Zbl 0947.03008. MR 1758510. DOI 10.1023/A:1004787622057. 446, 462

[24] Restall, G., "Multiple conclusions," pp. 189-205 in Logic, Methodology and Philosophy of Science (Oviedo, Spain, 2003), College Publications, London, 2005. 456

[25] Restall, G., "Truth values and proof theory," Studia Logica, vol. 92 (2009), pp. 241-64. Zbl 1191.03042. MR 2563170. DOI 10.1007/s11225-009-9197-y. 456

[26] Rousseau, G., "Sequents in many valued logic, I," Fundamenta Mathematicae, vol. 60 (1967), pp. 23-33. Zbl 0154.25504. MR 0210564. 458

[27] Rumfitt, I., "The categoricity problem and truth-value gaps," Analysis (Oxford), vol. 57 (1997), pp. 223-35. Zbl 0943.03586. MR 1482355. DOI 10.1111/1467-8284.00080. 459,464

[28] Rumfitt, I., “'Yes' and 'No,” Mind, vol. 109 (2000), pp. 781-823. MR 2131446. DOI 10.1093/mind/109.436.781. 447, 451, 455, 456

[29] Rumfitt, I., "Unilateralism disarmed: A reply to Dummett and Gibbard," Mind, vol. 111 (2002), pp. 305-21. MR 2133870. DOI 10.1093/mind/111.442.305. 463

[30] Rumfitt, I., "Co-ordination Principles: A Reply," Mind, vol. 117 (2008), pp. 1059-63. MR 2804681. DOI 10.1093/mind/fzn032. 463

[31] Rumfitt, I., "Knowledge by deduction," Grazer Philosophische Studien, vol. 77 (2008), pp. 61-84. 456

[32] Schroeder-Heister, P., "Generalized definitional reflection and the inversion principle," Logica Universalis, vol. 1 (2007), pp. 355-76. MR 2342688. DOI 10.1007/s11787-007-0018-7. 462

[33] Schröter, K., "Methoden zur Axiomatisierung beliebiger Aussagen- und Prädikatenkalküle," Zeitschrift für mathematische Logik und Grundlagen der Mathematik, vol. 1 (1955), pp. 241-51. MR 0077472. 458

[34] Shoesmith, D. J., and T. J. Smiley, Multiple-conclusion Logic, Cambridge Univ. Press, Cambridge, 1978. MR 0500331. 463

[35] Smiley, T., "Rejection," Analysis (Oxford), vol. 56 (1996), pp. 1-9. Zbl 0943.03606. MR 1379622. DOI 10.1111/j.0003-2638.1996.00001.x. 447, 451, 453, 454

[36] Stevenson, J. T., "Roundabout the runabout inference-ticket," Analysis, vol. 21 (1961), pp. 124-28. 446

[37] Tennant, N., Anti-realism and Logic: Truth as Eternal, Oxford Univ. Press, Oxford, 1987. 446

[38] Tennant, N., The Taming of the True, Oxford Univ. Press, Oxford, 1997. Zbl 0929.03001. MR 1469976. 446, 463

[39] Tennant, N., "Inferentialism, logicism, harmony and a counterpoint," in Essays for Crispin Wright: Logic, Language and Mathematics 2 vols. edited by A. Miller, Oxford Univ. Press, Oxford, forthcoming. 462

[40] Troelstra, A. S., and H. Schwichtenberg, Basic Proof Theory, 2nd ed., vol. 43 of Cambridge Tracts in Theoretical Computer Science, Cambridge Univ. Press, Cambridge, 2000. MR 1776976. DOI 10.1017/CBO9781139168717. 463

[41] Ungar, A. M., Normalization, Cut-elimination and the Theory of Proofs, vol. 28 of CSLI Lecture Notes, Stanford University Center for the Study of Language and Information, Stanford, 1992. Zbl 0866.03028. MR 1251962. 463 
[42] Wagner, S., “Tonk,” Notre Dame Journal of Formal Logic, vol. 22 (1981), pp. 289-300. Zbl 0442.03004. MR 0622360. 446

[43] Weir, A., "Classical harmony," Notre Dame Journal of Formal Logic, vol. 27 (1986), pp. 459-82. Zbl 0631.03003. MR 0866991. DOI 10.1305/ndjfl/1093636761. 446

\section{Acknowledgments}

This research is supported by the Alexander von Humboldt Foundation. I would also like to thank Pål Fjeldvig Antonsen, Denis Bonnay, Colin Caret, Aaron Cotnoir, Catarina Dutilh Novaes, Roy Dyckhoff, Hannes Leitgeb, Julien Murzi, Graham Priest, Stephen Read, Greg Restall, Ian Rumfitt, David Ripley, Stewart Shapiro, Peter Simons, and Elia Zardini for helpful feedback and discussion of earlier drafts. Finally, I'm immensely grateful to an anonymous referee for detailed suggestions on how to improve the paper.

Munich Center for Mathematical Philosophy (MCMP)

Ludwig-Maximilians-Universität München (LMU)

Chair of Logic and Philosophy of Language

Geschwister-Scholl-Platz 1

D-80539 Munich

Germany

ole.hjortland@Imu.de

http://Imu-munich.academia.edu/OleHjortland 